\title{
Ascorbate-Deficient vtc2 Mutants in Arabidopsis Do Not Exhibit Decreased Growth
}

\author{
Benson Lim ${ }^{1+}$, Nicholas Smirnoff ${ }^{2 \neq}$, Christopher S. Cobbett ${ }^{1}$ and John F. Golz ${ }^{1 *}$ \\ ${ }^{1}$ School of BioSciences, University of Melbourne, Parkville, VIC, Australia, ${ }^{2}$ Biosciences, College of Life and Environmental \\ Sciences, University of Exeter, Exeter, UK
}

In higher plants the L-galactose pathway represents the major route for ascorbate biosynthesis. The first committed step of this pathway is catalyzed by the enzyme GDP-L-galactose phosphorylase and is encoded by two paralogs in Arabidopsis VITAMIN C2 (VTC2) and VTC5. The first mutant of this enzyme, vtc2-1, isolated via an EMS mutagenesis screen, has approximately 20-30\% of wildtype ascorbate levels and has been reported to have decreased growth under standard laboratory conditions.

OPEN ACCESS

Edited by:

Irene Murgia,

Università degli Studi di Milano, Italy

Reviewed by:

Christine Helen Foyer,

University of Leeds, UK

Robert L. Last,

Michigan State University, USA

*Correspondence: John F. Golz

jgolz@unimelb.edu.au orcid.org/0000-0001-9478-5459

tPresent address: Benson Lim, Genome Institute of Singapore,

Genome, Singapore

forcid.org/0000-0001-5630-5602

Specialty section:

This article was submitted to Plant Physiology,

a section of the journal Frontiers in Plant Science

Received: 22 April 2016 Accepted: 28 June 2016 Published: 13 July 2016

Citation:

Lim B, Smirnoff N, Cobbett CS and Golz JF (2016) Ascorbate-Deficient vtc2 Mutants in Arabidopsis Do Not Exhibit Decreased Growth.

Front. Plant Sci. 7:1025. doi: 10.3389/fpls.2016.01025
Here, we show that a T-DNA insertion into the VTC2 causes a similar reduction in ascorbate levels, but does not greatly affect plant growth. Subsequent segregation analysis revealed the growth defects of vtc2-1 mutants segregate independently of the vtc2-1 mutation. These observations suggest that it is the presence of an independent cryptic mutation that affects growth of vtc2-1 mutants, and not the $70-80 \%$ decrease in ascorbate levels that has been assumed in past studies.

Keywords: Arabidopsis, ascorbate, VTC2, VTC5, vitamin C, GDP-L-galactose phosphorylase

\section{INTRODUCTION}

Ascorbate (vitamin C) is an important multifunctional antioxidant compound involved in stress tolerance (Conklin et al., 1996; Pastori et al., 2003; Sanmartin et al., 2003; Barth et al., 2004; Filkowski et al., 2004; Müller-Moulé et al., 2004; Chen and Gallie, 2005; Larkindale et al., 2005; Pavet et al., 2005) and redox signaling (Noctor and Foyer, 2011; Page et al., 2012). While several possible biosynthetic pathways for ascorbate have been proposed, it was demonstrated that the Lgalactose pathway is the dominant route in Arabidopsis (Dowdle et al., 2007). In this pathway, GDP-D-mannose, formed from D-mannose 1-phosphate, is sequentially converted to GDP-Lgalactose, L-galactose 1-phosphate, L-galactose, L-galactono-1,4-lactone, and finally to L-ascorbate. The first committed step of this pathway is catalyzed by GDP-L-galactose phosphorylase, which is encoded by the paralogous genes VITAMIN C2 (VTC2) and VTC5 and is most likely an important control point in the pathway (Yoshimura et al., 2014).

The first ascorbate-deficient mutants of Arabidopsis, $v t c 1-1$ and $v t c 2-1$, were identified via an ethyl-methanesulfonate (EMS) mutagenesis screen for ozone sensitive mutants (Conklin et al., 1996). Later, using a high-throughput nitroblue tetrazolium assay several more EMS-induced ascorbate-deficient mutants (vtc1-2, vtc2-2, vtc2-3, vtc3-1, vtc4-1) were identified (Conklin et al., 2000). Of these mutants, $v t c 1-1$ and $v t c 2-1$ have been extensively studied owing to their low levels

Abbreviations: ABRC, Arabidopsis Biological Resource Centre; EMS, Ethyl-Methanesulfonate; RT-PCR, Reverse Transcription Polymerase Chain Reaction; SAIL, Syngenta Arabidopsis Insertion Library. 
of ascorbate (Veljovic-Jovanovic et al., 2001; Jander et al., 2002; Pastori et al., 2003; Müller-Moulé et al., 2004; Pavet et al., 2005; Dowdle et al., 2007; Mukherjee et al., 2010; Kempinski et al., 2011; Kerchev et al., 2011; Lee et al., 2011; Luna et al., 2011; Talla et al., 2011; Zechmann, 2011; Botanga et al., 2012; Brosché and Kangasjärvi, 2012; Page et al., 2012; Zhang et al., 2012; Wang et al., 2013). The vtc1-1 mutant has a Pro22Ser substitution in the active site of GDP-mannose pyrophosphorylase (Conklin et al., 1999) resulting in a 70\% decrease in ascorbate levels (Pavet et al., 2005; Mukherjee et al., 2010; Kerchev et al., 2011; Zechmann, 2011; Zhang et al., 2012). vtc2-1 has a single base substitution ( $G$ to $A$ ) at the predicted 3 ' splice site of the fifth intron, resulting in an 80-90\% reduction in transcript levels and less than $2 \%$ of GDP-L-galactose phosphorylase activity in leaves (Dowdle et al., 2007). As a consequence, this mutant has 70-80\% reduction in ascorbate levels (Müller-Moulé et al., 2004; Pavet et al., 2005; Dowdle et al., 2007; Kerchev et al., 2011). Both mutant lines display reduced growth suggesting that ascorbate plays an important role in the control of plant growth (Veljovic-Jovanovic et al., 2001; Pastori et al., 2003; Müller-Moulé et al., 2004; Pavet et al., 2005; Kerchev et al., 2011). The other vtc2 mutants, vtc22 and $v t c 2-3$, have mis-sense mutations leading to Gly223Asp and Ser290Phe substitutions, respectively (Jander et al., 2002; Dowdle et al., 2007) and while the amount of ascorbate in these lines varies according to growth conditions, values typically range from $30-80 \%$ and $40-50 \%$ of wildtype levels (Conklin et al., 2000; Colville and Smirnoff, 2008). Interestingly, neither vtc2-2 nor $v t c 2-3$ display noticeable growth defects (Conklin et al., 2000).

Because vtc2-1 has detectable levels of full-length VTC2 transcript (Dowdle et al., 2007) and is thus not a complete lossof-function mutant, we obtained a T-DNA insertion mutant from the Arabidopsis stock center. We demonstrate that this mutant, here named $v t c 2-4$, lacks wildtype VTC2 transcripts and, although it has a level of ascorbate similar to vtc2-1, exhibits a near wildtype growth phenotype under short-day and continuous light conditions. We suggest that the decreased growth phenotype of $v t c 2-1$ is likely due to an independent cryptic mutation and is not due to ascorbate deficiency as widely assumed in the literature.

\section{MATERIALS AND METHODS}

\section{Plant Materials and Growth Conditions}

Mutant alleles were all in the Col-0 background and were sourced from the ABRC stock center. For growth assays under short days, seeds were sown on soil and placed in a growth chamber and exposed to an $8 \mathrm{~h}$ light (150-250 $\mu \mathrm{mol}$ photons $\left.\mathrm{m}^{-2} \mathrm{~s}^{-1}\right) / 16 \mathrm{~h}$ dark cycle at $21^{\circ} \mathrm{C}$ for 6 weeks. Plant weight was recorded and leaf surface area measurements taken by imagining freshly collected leaves and using ImageJ software to determine the surface area of leaf silhouettes. For growth under continuous light conditions, surface-sterilized seeds were germinated on agar media containing mineral salts (MM) with 2\% sucrose (MMS) and $0.8 \%$ Bacto agar as described by Scholl et al. (1998). Seedlings were grown at $20^{\circ} \mathrm{C}$ in a climate chamber with a $16 \mathrm{~h}$ light (150$250 \mu \mathrm{mol}$ photons $\left.\mathrm{m}^{-2} \mathrm{~s}^{-1}\right) / 8 \mathrm{~h}$ dark cycle for 7 days before transfer to soil in a climate chamber with $24 \mathrm{~h}$ continuous light (150-250 $\mu$ mol photons $\mathrm{m}^{-2} \mathrm{~s}^{-1}$ ).

For comparison of leaf area and chlorophyll fluorescence under short and long day conditions, plants were grown in pots ( $5 \mathrm{~cm}$ square and $5 \mathrm{~cm}$ deep) containing four parts Levington F2 compost (Scotts, Maryville, OH, USA) plus one part vermiculite. They were grown in short days $(8 \mathrm{~h})$ for 22 days after sowing in a controlled environment room at $23^{\circ} \mathrm{C}$ and $65 \%$ relative humidity with a light intensity of $200 \mu \mathrm{mol}$ photons $\mathrm{m}^{-2} \mathrm{~s}^{-1}$. After 22 days, half the plants were transferred to long days $(14 \mathrm{~h})$ under otherwise identical conditions and the leaf area and chlorophyll fluorescence measured over 10 days. The plants were imaged with a CF Imager (Technologica Ltd., Colchester, UK). They were dark adapted for $30 \mathrm{~min}$ before measuring basal fluorescence $\left(F_{0}\right)$. This was followed by measuring maximum dark adapted fluorescence $\left(F_{m}\right)$ resulting from a saturating light flash $\left(6,349 \mu \mathrm{mol}\right.$ photons $\mathrm{m}^{-2} \mathrm{~s}^{-1}$ for $\left.0.8 \mathrm{~s}\right)$. Darkadapted quantum efficiency of photosystem II was calculated as $F_{v} / F_{m}\left(F_{v}=F_{m}-F_{o}\right)$ (Baker, 2008). Leaf area of the imaged rosettes was extracted from the chlorophyll fluorescence images and the instrument was calibrated with leaf discs of known area.

\section{Measurement of Leaf Ascorbate Content in Seedlings}

Two-week old seedlings were harvested for ascorbate assay. Three seedlings from each control line (Col-0, single mutant lines) and 15-20vtc2-4;vtc5 seedlings were pooled for each biological replicate. Total ascorbate (ascorbate and dehydroascorbate) was measured by the iron (III) reduction assay (Kampfenkel et al., 1995) in an 8x scaled-down protocol.

\section{RNA Isolation and RT-PCR Analysis}

Total RNA from 3-week old shoot tissues was isolated using the Qiagen RNeasy Plant Mini Kit ${ }^{1}$ according to the manufacturer's protocol. The extracted RNA was further treated to remove any contaminating DNA using the DNA-free ${ }^{\mathrm{TM}} \mathrm{kit}^{2}$ according to the manufacturer's protocol. First strand cDNA was synthesized using the SuperScript ${ }^{\circledR}$ III First Strand Synthesis system ${ }^{2}$ according to the manufacturer's protocol. The full length VTC2 coding DNA sequence was amplified with the following primers: forward (5'-CAAAAGAGTTCCGACCGTTG-3') and reverse $\left(5^{\prime}\right.$-ACTGAAGGACAAGGCACTCG-3'). The transcript of the constitutively expressed ACTIN2 (ACT2) was used as an internal control with the following primers: forward (5'-GGTAACATTGTGCTCAGTGGTGG-3') and reverse ( $5^{\prime}$ CTCGGCCTTGCAGATCCACATC-3' ${ }^{\prime}$ ). The PCR was carried out using Promega GoTaq $^{\circledR}$ Green Master $\mathrm{Mix}^{3}$ with the following conditions: initial denaturation $5 \mathrm{~min}$ at $94^{\circ} \mathrm{C}$ was followed by 30 cycles of $30 \mathrm{~s}$ denaturation step at $94^{\circ} \mathrm{C}, 30 \mathrm{~s}$ annealing step at $60^{\circ} \mathrm{C}$ and $60 \mathrm{~s}$ extension step at $72^{\circ} \mathrm{C}$. The final extension step was for $5 \mathrm{~min}$ at $72^{\circ} \mathrm{C}$.

\footnotetext{
${ }^{1}$ www.qiagen.com

${ }^{2}$ www.lifetechnologies.com

${ }^{3}$ www.promega.com
} 


\section{Genotyping the vtc Mutants}

Plants were genotyped by PCR using DNA extracted with the preparation method described in Edwards et al. (1991). The $v t c 2-1, v t c 5-1$, and $v t c 5-2$ mutants were genotyped as described by Dowdle et al. (2007). The SAIL line of $v t c 2-4$ was genotyped by performing PCR using a triplet of primers; two primers complementary to genomic DNA sequences situated on either side of the insertion site and a third primer complementary to the left border of the vector pDAP101. Primer sequences were as follows: forward (5'-TGATAATGGTTTCTGTAGCTTGGA-3'), reverse (5'-AAAACCAAGCTCTCTGCACAA- $\left.3^{\prime}\right)$ and LB1 (5'-GCCTTTTCAGAAATGGATAAATAGCCTTGCTTCC - 3'). Accession Numbers $v t c 2-4$ and $v t c 2-1 W$ (line\#4) have been deposited with ABRC and given the stock numbers CS69540 and CS69541, respectively.

\section{RESULTS}

\section{Genetic Characterization of a vtc2 T-DNA Insertion Mutant}

A line predicted to have a T-DNA insertion in VTC2 (SAIL_769_H05/CS876707) was obtained from the Arabidopsis stock center (ABRC). PCR genotyping and sequencing of genomic DNA adjacent to the T-DNA left border confirmed the presence of the T-DNA within the fourth exon of VTC2 at position +620 downstream of the start codon $(+1)$. Lines homozygous for this insertion mutation, hereafter called $v t c 2-$ 4, were backcrossed to wildtype (Col-0) and the resulting F2 progeny PCR genotyped for presence of the T-DNA allele. Numbers of VTC2/VTC2:vtc2-4/VTC2:vtc2-4/vtc2-4 plants were consistent with an expected 1:2:1 segregation ratio (48:112:56; $\left.\chi^{2}=0.89 ; p>0.6\right)$. Subsequent RT-PCR analysis failed to amplify a full-length VTC2 transcript from lines homozygous for $v t c 2-4$ confirming presence of the T-DNA within this gene (Supplementary Figure S1). Measurement of total ascorbate in leaves of 4-week-old wildtype, $v t c 2-1$ and $v t c 2-4$ plants revealed that both $v t c 2-4$ and $v t c 2-1$ had approximately $30 \%$ the levels of ascorbate that are found in wildtype, showing that the T-DNA present in the vtc2-4 line conditions a similar decrease in ascorbate (Figure 1A).

Previous studies have shown that the EMS-induced mutant $v t c 2-1$ has a growth defect (Müller-Moulé et al., 2004; Pavet et al., 2005; Kerchev et al., 2011). However, when vtc2-4 mutants were propagated on soil under continuous light, they had a wildtype appearance (Figure 1B) and fresh weight measurements differed little from wildtype (Figure 1C). In contrast, vtc2-1 mutants were significantly smaller than $v t c 2-4$ mutants, with approximately half the fresh weight of wildtype. To ensure that the observed growth difference between $v t c 2-1$ and $v t c 2$ 4 mutants are a general feature of these mutants and not just induced by long-days, leaf surface area measurements were assessed over a 10-day growth period under both short and long-days conditions. Regardless of day length, $v t c 2-4$ mutants had a marginally smaller leaf surface area when compared to wildtype, whereas $v t c 2-1$ mutants were considerably smaller than

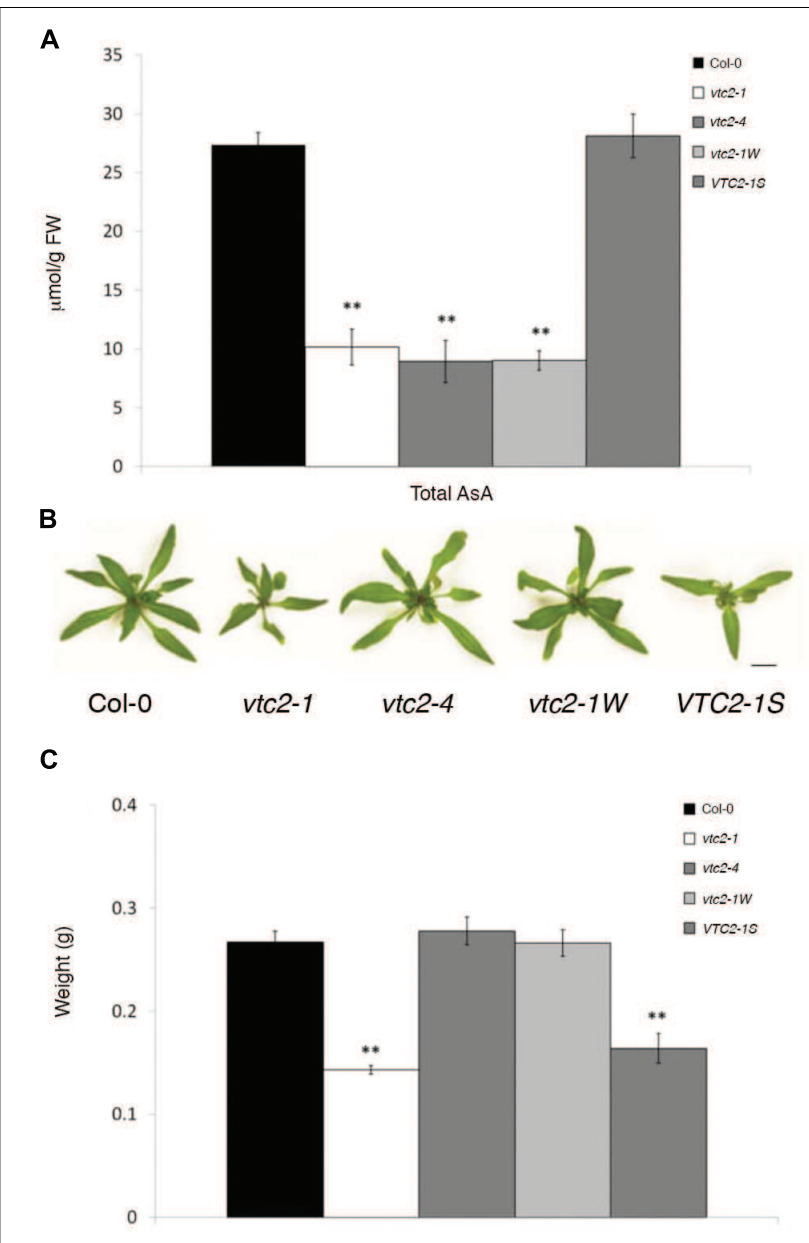

FIGURE 1 | (A-C) Leaf ascorbate concentration and fresh weights of Arabidopsis thaliana vtc2 mutants. (A) Total ascorbate (ascorbate and dehydroascorbate) concentration in leaves of 4-week-old plants. Error bars indicate SE $(n=3)$. Significant differences from Col-0 wildtype were determined with Student's $t$-test $(* * p<0.01)$. Significance values were adjusted for multiple comparisons using the Bonferroni correction. (B) Three-week-old Col-0 wildtype, vtc2-1, vtc2-4, vtc2-1W, VTC2-1S grown under continuous light. Scale bar $=10 \mathrm{~mm}$. (C) Shoot fresh weight measurements of 3-week-old plants grown under continuous light conditions. Error bars indicate SE $(n=6)$. Significant differences from Col-0 wildtype were determined with Student's $t$-test $(* * p<0.01)$. Significance values were adjusted for multiple comparisons using the Bonferroni correction.

wildtype and $v t c 2-4$ mutants (Figures 2A,B). A multi-factor ANOVA test confirmed that differences in leaf size between $v t c$ mutant lines and wildtype under either growth condition was significant $(p<0.0001$; Table 1). To determine whether the observed difference in leaf surface area arises from altered rates of growth, relative leaf expansion rate of rosettes under long and short day conditions were calculated. As expected, the growth rate was largely influenced by day length as each line displayed significantly higher growth under long days (Figure 2C). In contrast, there was no significant difference in the growth rate between wildtype and the $v t c 2$ mutants under long days. While this was also true for wildtype and $v t c 2-4$ 


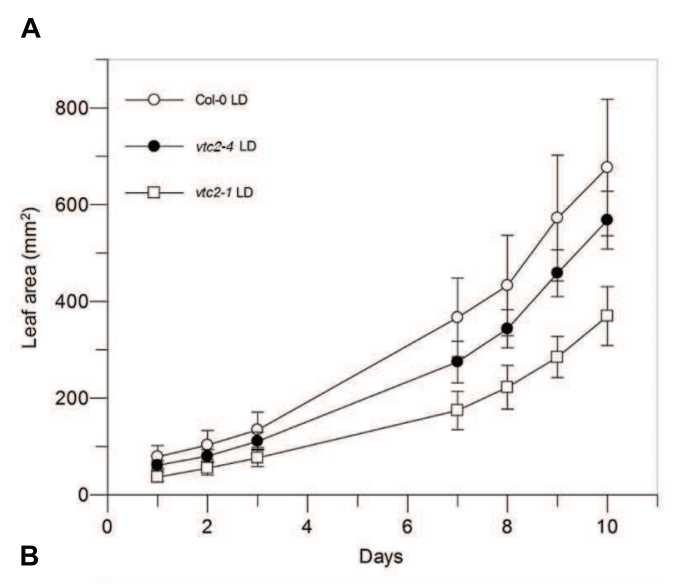

D

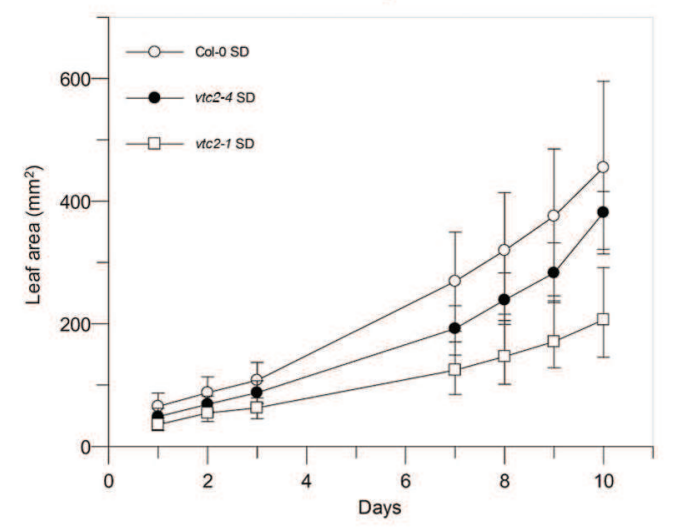

$v t c 2-4$

Col-0<smiles>C[12CH3]</smiles>

vtc2-1

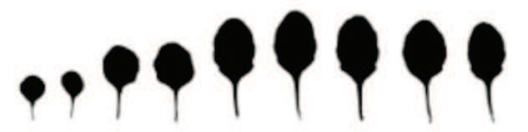

VTC2-1S<smiles>C[13CH3]</smiles>

$v t c 2-1 W$

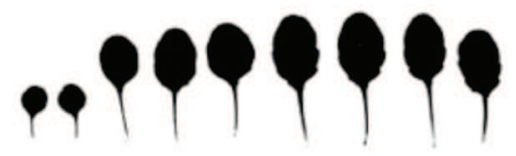

C

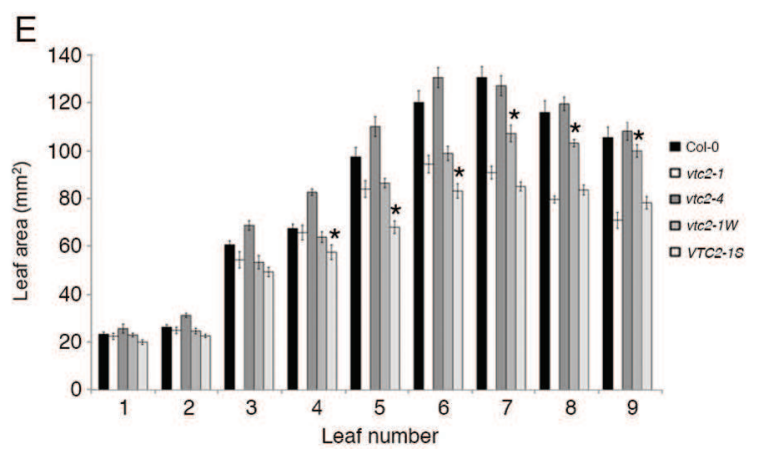

FIGURE 2 | (A-C) Rosette and leaf area measurements of Arabidopsis thaliana vtc2 mutants. (A) Surface area measurements $\left(\mathrm{mm}^{2}\right)$ of leaves taken over a 10-day period under long-day (14 h) conditions. Values are mean and error bars indicate SD $(n=12)$. (B) Surface area measurements $\left(\mathrm{mm}^{2}\right)$ of leaves taken over a 10-day period under short-day $(8 \mathrm{~h})$ conditions. Values are mean and error bars indicate $\mathrm{SD}(n=12)$. (C) Relative leaf expansion rate $\left(\mathrm{RLER}\right.$, day $\left.{ }^{-1}\right)$ was calculated as the slope of a linear fit to a graph of natural log leaf area $(\mathrm{mm})$ versus time (days). RLER was calculated from data in Figures $\mathbf{2 A}$, $\mathbf{B}$. Values are mean and error bars indicate SD $(n=10-12)$. Significant differences were determined with a one-way ANOVA and Tukey HSD post hoc test $\left({ }^{*} p=0.005,{ }^{* *} p<0.001\right)$.

(D) Silhouettes of leaves from 6-week-old plants grown under short-day conditions. Scale bar $=1 \mathrm{~cm}$. (E) Surface area measurements $\left(\mathrm{mm}^{2}\right)$ of leaves taken from 6 -week-old plants grown under short-day conditions. Values are mean and error bars indicate SD $(n=7-10)$. Differences between leaf areas of vtc2-1, vtc2-1W and VTC2-1S were assessed using a Student's t-test $\left(^{*} p<0.05\right)$.

mutants under short days, vtc2-1 mutants had a significantly reduced growth rate when compared to wildtype (Figure 2C). Finding a smaller rosette leaf area for both $v t c 2-1$ and $v t c 2-4$ in long days (Figure 2A), but similar relative expansion rate to wildtype (Figure 2C), implies that the small size results from initially smaller seedlings rather than from differences in intrinsic leaf expansion rate. However, under short-day conditions, $v t c 2-1$ relative leaf expansion rate is compromised as well. Measuring the surface area of individual leaves collected from 6-week old short-day grown plants revealed that the size differences between wildtype and $v t c 2-1$ mutants is mostly confined to the fifth and subsequent leaves (Figures 2D,E). Taken together these data show that the growth defects of $v t c 2$ 4 mutants are not of the same magnitude as those seen in 
TABLE 1 | A comparison of the rosette leaf areas of wildtype (Col-0) and vtc2 mutants of Arabidopsis thaliana using multivariate ANOVA.

\begin{tabular}{lcc}
\hline Test & Condition & $\boldsymbol{p}$-value \\
\hline Col-0 vs. vtc2-4 & LD & 0.0089 \\
Col-0 vs. vtc2-1 & LD & $<0.0001$ \\
vtc2-4 vs. vtc2-1 & LD & $<0.0001$ \\
Col-0 vs. vtc2-4 & SD & $<0.0001$ \\
Col-0 vs. vtc2-1 & SD & $<0.0001$ \\
vtc2-4 vs. vtc2-1 & SD & $<0.0001$ \\
\hline
\end{tabular}

The leaf area data are shown in Figures $2 A, B$.

$v t c 2-1$ and hence may not be directly attributable to ascorbate deficiency.

\section{The Small Size Phenotype of vtc2-1 Is Not Linked to Ascorbate Deficiency}

Since $v t c 2-1$ and $v t c 2-4$ have contrasting growth phenotypes, we undertook a genetic characterization of $v t c 2-1$. From a cross between wildtype and vtc2-1 F1 individuals, heterozygous for $v t c 2-1$, exhibited wildtype growth in soil under continuous light condition (data not shown). The presence of the vtc2-1 allele can be detected by CAPS (cleaved amplified polymorphic sequence) PCR (Dowdle et al., 2007). In a segregating F2 population grown in soil, genotyping confirmed that the vtc21 allele segregated in a Mendelian ratio (Table $2 ; \chi^{2}=2.23$; $p>0.3$ ). Four-week-old individual F2 plants were also visually scored for size as "Wildtype" or "Small." While wildtype and vtc21 mutants grown in parallel showed a clear difference in size as previously reported (Veljovic-Jovanovic et al., 2001; MüllerMoulé et al., 2004; Pavet et al., 2005; Kerchev et al., 2011), some individual wildtype plants exhibited a similar growth size to vtc2-1 mutant plants. However, an excess of small individuals over the expected number suggested it was likely that some wildtype individuals were misclassified as "Small." A number of individuals with a small growth phenotype were homozygous for the wildtype VTC2 allele and, conversely, some vtc2-1 homozygous individuals had a wildtype appearance (Table 2).

To score the growth phenotype more accurately a number of F2 lines derived from the Col-0/vtc2-1 cross were allowed to self-fertilize to produce individual F3 families, which were then observed as populations after 4-week-growth in soil under continuous light conditions (Table 3). Of seven F2 individuals wildtype at the VTC2 locus with an apparent small growth phenotype, five F3 families were uniformly small in size (called VTC2-1S; Figure 1B). Conversely, of 24 F2 individuals homozygous for $v t c 2-1$ and apparently wildtype in

TABLE 2 | Phenotypic scoring of 4-week-old F2 individuals from a cross between wildtype (Col-0) and vtc2-1 Arabidopsis thaliana mutants.

\begin{tabular}{lcccc}
\hline Phenotype & VTC2/VTC2 & VTC2/vtc2-1 & vtc2-1/vtc2-1 & Total \\
\hline Small & 12 & 34 & 44 & 90 \\
Wildtype & 60 & 126 & 31 & 217 \\
Total & 72 & 160 & 75 &
\end{tabular}

TABLE 3 | Growth phenotypes of 4-week-old F3 individuals.

\begin{tabular}{lcc}
\hline $\begin{array}{l}\text { Phenotype and } \\
\text { genotype of F2 line }\end{array}$ & $\begin{array}{c}\text { Growth phenotypes } \\
\text { segregating }\end{array}$ & $\begin{array}{c}\text { Growth phenotypes } \\
\text { uniform }\end{array}$ \\
\hline $\begin{array}{l}\text { Small }- \text { VTC2NVTC2 } \\
\text { (VTC2-1S) }\end{array}$ & 2 & $5^{\mathrm{a}}$ \\
$\begin{array}{l}\text { Wildtype }- \text { vtc2/vtc2 } \\
\text { (vtc2-1W) }\end{array}$ & 6 & $18^{\mathrm{b}}$
\end{tabular}

Seeds derived from F2 lines were germinated on agar media under long day conditions for 7 days before transfer to soil under continuous light. Growth phenotype of 4-week-old F3 individuals were scored relative to $\mathrm{Col}-\mathrm{O}$ and vtc21 plants grown in parallel.

a All plants have a small growth phenotype. ${ }^{\mathrm{b}}$ All plants have a wildtype growth phenotype.

size, 18 F3 families were uniformly wildtype in size (called $v t c 2$ $1 W$; Figure 1B). Measuring fresh weight and total ascorbate levels under continuous light conditions revealed that $v t c 2-1 \mathrm{~W}$ plants had a similar weight to wildtype, but with ascorbate levels decreased by approximately $70 \%$ compared to wildtype. Likewise, fresh weight of a representative VTC2-1S line was similar to $v t c 2-1$, but with ascorbate approaching wildtype levels (Figure 1C).

To better characterize the growth characteristics of these F3 lines, fresh weight and leaf surface area measurements of representative $v t c 2-1 W$ and VTC2-1S plants grown under short-day were made (Figures 2D,E, Supplementary Figure S2). In agreement with the measurements made under continuous light conditions, the fresh weight of the vtc2-1W line was statistically different from $v t c 2-1$ mutant controls (Supplementary Figure S2). When compared to $v t c 2-1$ mutants, late arising leaves of $v t c 2-1 W$ plants were larger (leaf 7 to leaf 9; increase ranged from 18 to $41 \%$ ). In contrast, the fresh weight of a representative VTC2-1S line was not significantly different from $v t c 2-1$ mutants (Supplementary Figure S2). This is despite finding that a few VTC2-1S leaves were slightly smaller than those of $v t c 2-1$ (leaf 5-6; decrease ranged from 13 to 23\%). In summary, these analyses demonstrate that ascorbate-deficiency is genetically separable from growth defects associated with the $v t c 2-1$ mutant line.

\section{Leaf Senescence in vtc2 Mutants}

Given that ascorbate deficiency in $v t c 2-4$ mutants is not associated with a strong growth defect, we next considered whether this line displays other ascorbate-deficient characteristics. Previous studies have shown that reduced ascorbate is associated with faster leaf senescence (Barth et al., 2004). Senescence can be measured as a loss of leaf photosynthetic efficiency. Thus to assess the extent of leaf senescence in $v t c 2$ mutants, we imaged dark-adapted quantum efficiency of photosystem II $\left(F_{v} / F_{m}\right)$. Examining the $F_{v} / F_{m}$ images of wildtype, $v t c 2-4$ and $v t c 2-1$ grown under both short and long days suggested bigger decreases in older leaves of $v t c 2$ mutants compared to wildtype (Figure 3A). To quantify the different rates of senescence in each line, the \% of leaf area below a fixed $F_{v} / F_{m}$ value ( 0.75$)$ over days $7-10$ was calculated. This revealed a slight elevation in the rate of senescence under short days compared to long days for all lines tested which was only significant for $v t c 2-4$ 


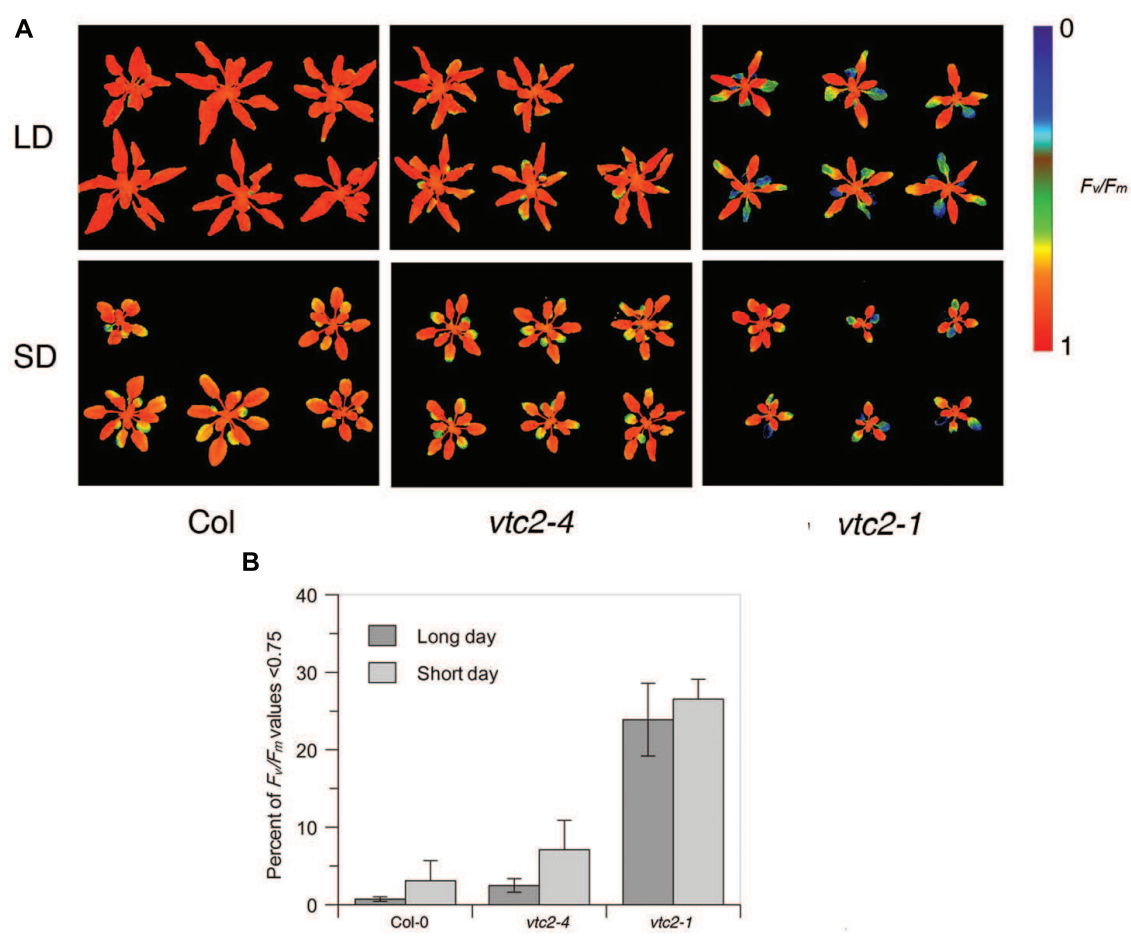

FIGURE 3 | (A,B) $\boldsymbol{F}_{\boldsymbol{v}} / \boldsymbol{F}_{\boldsymbol{m}}$ measurements of Arabidopsis thaliana $\boldsymbol{v} \boldsymbol{t c 2}$ mutants. (A) Chlorophyll fluorescence images of short-day (SD) and long-day (LD) grown plants colored according to their $F_{v} / F_{m}$ ratio on day 10 (see Figures $\mathbf{2 A}, \mathbf{B}$ ). (B) Percentage of leaf surface area with $F_{v} / F_{m}$ values below 0.75 in short-day and long-day grown plants. Data are pooled from days 7-10 (see Figures 2A,B). Error bars indicate SD $(n=6-12)$. ANOVA shows significant differences $(p<0.001)$ between Col-0 and the vtc2-1 mutant, whereas daylength only has a significant effect on vtc2-4 mutant plants $(p<0.05)$.

$(p<0.05)$ (Figure 3B). However, under both growth conditions, $v t c 2-1$ mutants displayed a noticeable and statistically significant $(p<0.001)$ increase in the proportion of $F_{v} / F_{m}$ values below 0.75 when compared to wildtype, whereas $v t c 2-4$ mutants only exhibit a marginal and statistically non-significant increase (Figure 3B). These observations are consistent with $v t c 2-1$ mutants displaying increased leaf senescence, which is not apparent in $v t c 2-4$ under these growth conditions.

\section{Arabidopsis Lacking Functional GDP-L-galactose Phosphorylase has Undetectable Ascorbate and Exhibits a Seedling-Lethal Phenotype}

The importance of the L-ascorbate biosynthetic pathway for Arabidopsis growth and development was demonstrated by Dowdle et al. (2007) when they showed that $v t c 2-1 ; v t c 5$ double mutants displayed a growth arrest following germination; a phenotype that was also associated with cotyledon bleaching. Given that $v t c 2-1$ is not a complete loss-of-function allele and also apparently harbors a cryptic mutation/s that affect growth, we reconstituted the vtc2;vtc5 double mutant line by combining the $v t c 2-4$ mutant with two different $v t c 5$ mutant alleles ( $v t c 5-1$ and $v t c 5-2$; Dowdle et al., 2007). The resulting $v t c 2-4 ; v t c 5-1$ and $v t c 2-4 ; v t c 5-2$ double mutants also exhibited a seedling-lethal phenotype, and cotyledons were subsequently bleached upon germination (Figure 4A). These were phenotypically indistinguishable from the $v t c 2-1 ; v t c 5$ double mutants previously described (Dowdle et al., 2007). Consistent with the previous study, both double mutants had undetectable levels of ascorbate (Figure 4B). The double mutants could be rescued to complete their life cycles by supplementation with ascorbate (Figure 4C). Thus these experiments show that the phenotype of the double mutants between $v t c 2-1$ and $v t c 5$ produced by Dowdle et al. (2007) is not caused by additional mutations in $v t c 2-1$ and confirm the importance of the L-ascorbate biosynthetic pathway for seedling viability.

\section{DISCUSSION}

It has been widely reported that Arabidopsis mutants with severe ascorbate deficiency exhibit a small growth phenotype and that this decreased growth is thus casually linked to ascorbate deficiency (Jander et al., 2002; Müller-Moulé et al., 2004; Pavet et al., 2005; Dowdle et al., 2007; Giacomelli et al., 2007; Kerchev et al., 2011). Here we show that the decreased growth phenotype of the vtc2-1 mutant line is not linked to ascorbate deficiency using two approaches; (1) the vtc2-4 mutant, an apparent null mutant which has comparable levels of ascorbate to $v t c 2-1$, shows a different growth profile when compared to $v t c 2-1$ mutants; (2) after backcrossing $v t c 2-1$ to wildtype plants we readily isolated $v t c 2-1$ ascorbate-deficient lines with weight and leaf sizes that 


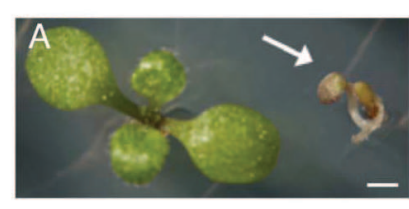

Col-0
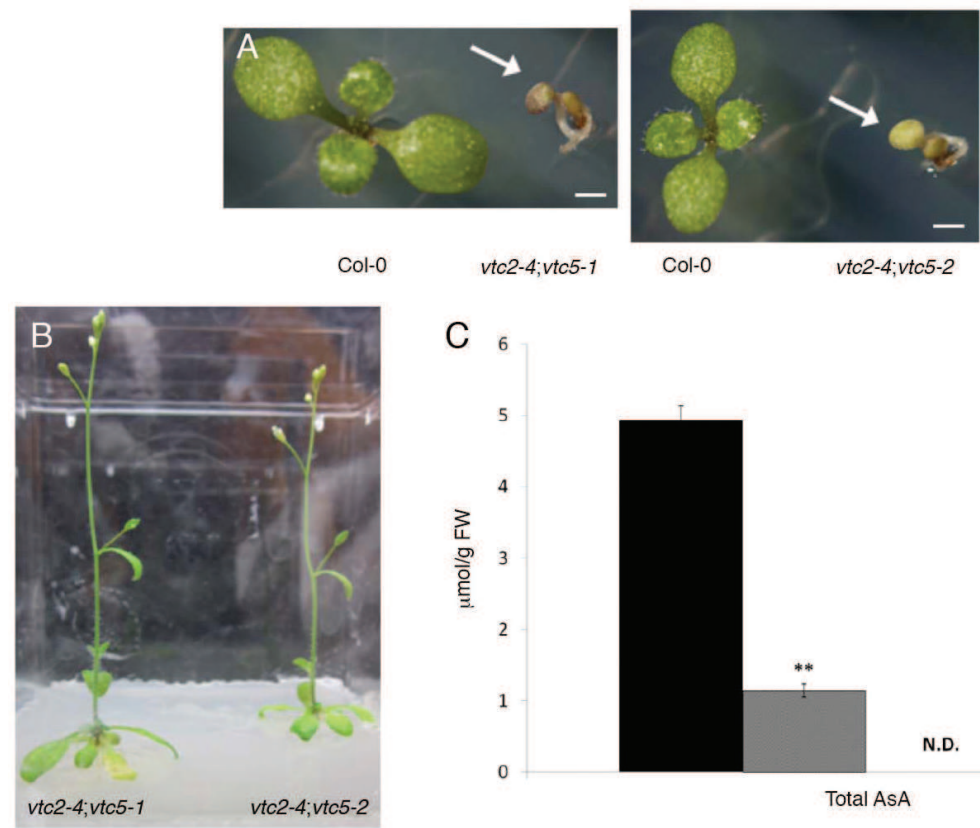

C 6

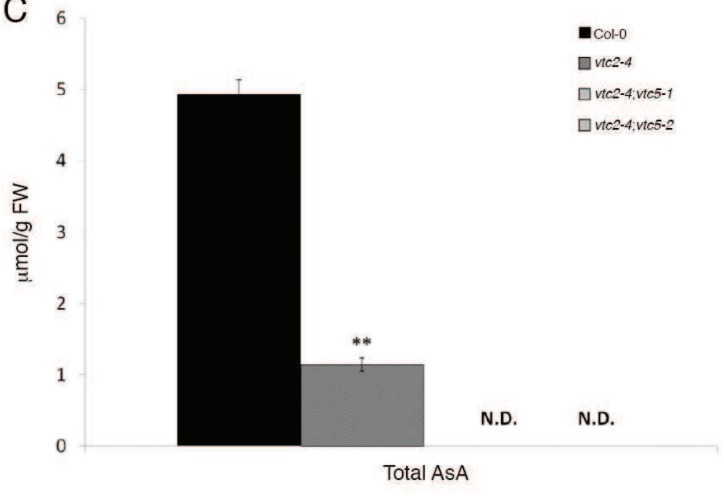

FIGURE 4 | (A-C) Growth and leaf ascorbate concentration of Arabidopsis thaliana vtc2-4;vtc5 double mutants. (A) One-week old Col-0 and vtc2-4; vtc5-1 double mutants (arrow) grown on agar media. Bar $=1 \mathrm{~mm}$. (B) Three-week-old vtc2-4;vtc5-1 double mutants grown on agar media supplemented with 0.5 mM L-ascorbic acid. (C) Total ascorbate (ascorbate and dehydroascorbate) concentration in leaves of 2-week-old seedlings grown on agar medium. Three seedlings from Col-0 and vtc2-4 and 15-20 vtc2-4;vtc5 seedlings were pooled for each sample. Error bars indicate SE $(n=3)$. Significant differences from Col-0 wildtype were determined with Student's $t$-test with significance values adjusted for multiple comparisons using the Bonferroni correction $(* * p<0.01)$.

are closer to wildtype than $v t c 2-1$ mutants, as well as lines with a small growth phenotype that were homozygous for VTC2. It appears that the decreased growth phenotype of the $v t c 2-1$ line is caused by an additional mutation/s that have likely arisen during the original EMS mutagenesis process (Conklin et al., 1996). Because of the difficulty of accurately scoring the size of individual F2 plants it is not clear from our analysis whether the $v t c 2$ mutation and those associated with the growth defect are linked. However, it is unlikely that they are closely linked because it was relatively easy to identify F2 individuals homozygous for the wildtype allele at one locus but homozygous mutant at the other.

The vtc1-1 mutant (Conklin et al., 1996) also exhibits a decreased growth phenotype (Veljovic-Jovanovic et al., 2001; Pavet et al., 2005; Kerchev et al., 2011; Talla et al., 2011). VTC1 produces GDP-mannose, which has important functions in cell wall carbohydrate biosynthesis and protein glycosylation in addition to ascorbate biosynthesis (Conklin et al., 1999; Lukowitz et al., 2001). It is therefore possible that deficiency of GDPmannose results in a number of different biochemical defects leading to a small growth phenotype that is not specifically due to ascorbate deficiency.

The vtc2-1 mutant line has been extensively analyzed (Müller-Moulé et al., 2003; Mukherjee et al., 2010; Gao et al., 2011; Zechmann, 2011; Botanga et al., 2012; Page et al., 2012) and has also been used to generate different mutant combinations in a number of studies. For example, interactions between redox metabolism and abscisic acid signaling were investigated by crossing vtc2-1 with an abscisic acid insensitive mutant (abi4). The vtc2-1;abi4 double mutant that was subsequently characterized, exhibited wildtype growth leading to the suggestion that abi4 suppressed the growth phenotype of $v t c 2-1$ (Kerchev et al., 2011). It is plausible that from the cross, the vtc2-1;abi4 double mutant selected for analysis lacked the cryptic mutation(s) conferring the small growth phenotype. Furthermore, the use of whole genome microarray analysis to characterize differences between vtc21 and vtc2-1 abi4 mutant lines is likely to have been complicated by the possible absence of the cryptic mutation(s) in the double mutant background (Kerchev et al., 2011). The decreased growth phenotype of $v t c 2-1$ has also been described in combination with mutations affecting chronic photo-oxidative stress (Müller-Moulé et al., 2004), chloroplastic ascorbate peroxidase (Giacomelli et al., 2007) and the autoimmune response (Zhu et al., 2013).

Our data indicate that the ascorbate deficiency of $v t c 2$ mutants does not cause a large decrease in growth under laboratory conditions. While the $v t c 2-4$ has a slightly smaller rosette area than wildtype, relative leaf expansion rate is identical. It is likely that $v t c 2-4$ seedlings have smaller initial seedling size or vigor resulting in a magnifying size difference as the plants grow. Since the fully ascorbate deficient seedlings of the vtc2;vtc5 double mutant are not viable, it is apparent that a reduction greater than the $\sim 80 \%$ occurring in the $v t c 2-4$ mutant is needed to impact growth. Ascorbate is amongst the most abundant of primary metabolites in Arabidopsis leaves (compare the widely 
reported values of $2-20 \mu \mathrm{mol} \mathrm{g}^{-1}$ fresh weight for ascorbate with the most abundant sugars, organic acids and amino acids reported in Szecowka et al., 2013). The presence of ascorbate concentrations in leaves that are much greater than needed to support growth in laboratory conditions emphasizes the importance of its protective functions for plants growing in stressful or fluctuating natural environments (Munné-Bosch et al., 2013).

\section{CONCLUSION}

In summary, we suggest that the interpretation of some studies of the commonly used $v t c 2-1$ line or its derivatives may need to be re-evaluated. It is also important to take into consideration that the apparent cryptic mutation(s) affecting growth of $v t c 2-1$ may have a significant impact on physiological status and gene expression unrelated to ascorbate deficiency, so although it was critical in identifying its biosynthetic pathway, it is not suitable for investigating the wider functions of ascorbate.

\section{AUTHOR CONTRIBUTIONS}

BL, NS, and JG designed and performed the experiments, analyzed the data and drafted the manuscript. CC conceived the

\section{REFERENCES}

Baker, N. R. (2008). Chlorophyll fluorescence: a probe of photosynthesis in vivo. Ann. Rev. Plant Biol. 59, 89-113. doi: 10.1146/annurev.arplant. 59.032607.092759

Barth, C., Moeder, W., Klessig, D. F., and Conklin, P. L. (2004). The timing of senescence and response to pathogens is altered in the ascorbatedeficient Arabidopsis mutant vitamin c-1. Plant Physiol. 134, 1784-1792. doi: 10.1104/pp.103.032185

Botanga, C. J., Bethke, G., Chen, Z., Gallie, D. R., Feihn, O., and Glazebrook, J. (2012). Metabolite profiling of Arabidopsis inoculated with Alternaria brassicicola reveals that ascorbate reduces disease severity. Mol. Plant Microbe Interact. 25, 1628-1638. doi: 10.1094/MPMI-07-12-0179-R

Brosché, M., and Kangasjärvi, J. (2012). Low antioxidant concentrations impact on multiple signalling pathways in Arabidopsis thaliana partly through NPR1. J. Exp. Bot. 63, 1849-1861. doi: 10.1093/jxb/err358

Chen, Z., and Gallie, D. R. (2005). Increasing tolerance to ozone by elevating foliar ascorbic acid confers greater protection against ozone than increasing avoidance. Plant Physiol. 138, 1673-1689. doi: 10.1104/pp.105. 062000

Colville, L., and Smirnoff, N. (2008). Antioxidant status, peroxidase activity, and PR protein transcript levels in ascorbate-deficient Arabidopsis thaliana vtc mutants. J. Exp. Bot. 59, 3857-3868. doi: 10.1093/jxb/ern229

Conklin, P. L., Norris, S. R., Wheeler, G. L., Williams, E. H., Smirnoff, N., and Last, R. L. (1999). Genetic evidence for the role of GDP-mannose in plant ascorbic acid (vitamin C) biosynthesis. Proc. Natl. Acad. Sci. U.S.A. 96, 4198-4203. doi: 10.1073/pnas.96.7.4198

Conklin, P. L., Saracco, S. A., Norris, S. R., and Last, R. L. (2000). Identification of ascorbic acid-deficient Arabidopsis thaliana mutants. Genetics 154, 847-856.

Conklin, P. L., Williams, E. H., and Last, R. L. (1996). Environmental stress sensitivity of an ascorbic acid-deficient Arabidopsis mutant. Proc. Natl. Acad. Sci. U.S.A. 93, 9970-9974. doi: 10.1073/pnas.93.18.9970

Dowdle, J., Ishikawa, T., Gatzek, S., Rolinski, S., and Smirnoff, N. (2007). Two genes in Arabidopsis thaliana encoding GDP-L-galactose phosphorylase are study and its design and coordination, and assisted with revisions of the manuscript. All authors read and consented to the final version of the manuscript.

\section{ACKNOWLEDGMENTS}

This research is supported by a grant from the Australian Research Council (BL, CSC, JFG) and a Biotechnology and Biological Sciences Research Council grant (NS; BB/G021678/1).

\section{SUPPLEMENTARY MATERIAL}

The Supplementary Material for this article can be found online at: http://journal.frontiersin.org/article/10.3389/fpls.2016.01025

FIGURE S1 | Reverse transcription polymerase chain reaction analysis of the vtc2-4 mutant. Total RNA was extracted from 10-day old seedlings and RT-PCR was performed using VTC2 specific primers (top panel) that flank the full-length transcript. ACTIN2 (ACT2) specific primers were used as an internal control (bottom panel).

FIGURE S2 | Fresh weight measurements of short-day grown plants. Shoot fresh weights of indicated lines were assessed after 6-week growth under short-day conditions. Error bars indicate SE $(n=7-10)$. Significant differences were determined using a Student's $t$-test and statistical differences $(p<0.01)$ from Col-0 wildtype indicated with "a," whereas "b" denotes differences from vtc2-1 mutants.

required for ascorbate biosynthesis and seedling viability. Plant J. 52, 673-689. doi: 10.1111/j.1365-313X.2007.03266.x

Edwards, K., Johnstone, C., and Thompson, C. (1991). A simple and rapid method for the preparation of plant genomic DNA for PCR analysis. Nucleic Acids Res. 19:1349.

Filkowski, J., Kovalchuk, O., and Kovalchuk, I. (2004). Genome stability of vtc1, tt4, and tt5 Arabidopsis thaliana mutants impaired in protection against oxidative stress. Plant J. 38, 60-69. doi: 10.1111/j.1365-313X.2004.02020.x

Gao, Y., Badejo, A. A., Shibata, H., Sawa, Y., Maruta, T., Shigeoka, S., et al. (2011). Expression analysis of the VTC2 and VTC5 genes encoding GDP-L-galactose phosphorylase, an enzyme involved in ascorbate biosynthesis, in Arabidopsis thaliana. Biosci. Biotechnol. Biochem. 75, 1783-1788. doi: 10.1271/bbb.110320

Giacomelli, L., Masi, A., Ripoll, D. R., Lee, M. J., and van Wijk, K. J. (2007). Arabidopsis thaliana deficient in two chloroplast ascorbate peroxidases shows accelerated light-induced necrosis when levels of cellular ascorbate are low. Plant Mol. Biol. 65, 627-644. doi: 10.1007/s11103-007-9227-y

Jander, G., Norris, S. R., Rounsley, S. D., Bush, D. F., Levin, I. M., and Last, R. L. (2002). Arabidopsis map-based cloning in the post-genome era. Plant Physiol. 129, 440-450. doi: 10.1104/pp.003533

Kampfenkel, K., Kushnir, S., Babiychuk, E., Inzé, D., and Van Montagu, M. (1995). Molecular characterization of a putative Arabidopsis thaliana copper transporter and its yeast homologue. J. Biol. Chem. 270, 28479-28486. doi: 10.1074/jbc.270.47.28479

Kempinski, C. F., Haffar, R., and Barth, C. (2011). Toward the mechanism of NH4+ sensitivity mediated by Arabidopsis GDP-mannose pyrophosphorylase. Plant Cell Environ. 34, 847-858. doi: 10.1111/j.1365-3040.2011. 02290.x

Kerchev, P. I., Pellny, T. K., Vivancos, P. D., Kiddle, G., Hedden, P., Driscoll, S., et al. (2011). The transcription factor ABI4 is required for the ascorbic acid-dependent regulation of growth and regulation of jasmonate-dependent defense signaling pathways in Arabidopsis. Plant Cell 23, 3319-3334. doi: 10.1105/tpc.111.090100

Larkindale, J., Hall, J. D., Knight, M. R., and Vierling, E. (2005). Heat stress phenotypes of Arabidopsis mutants implicate multiple signaling pathways 
in the acquisition of thermotolerance. Plant Physiol. 138, 882-897. doi: 10.1104/pp.105.062257

Lee, Y., Park, C. H., Kim, A. R., Chang, S. C., Kim, S.-H., Lee, W. S., et al. (2011). The effect of ascorbic acid and dehydroascorbic acid on the root gravitropic response in Arabidopsis thaliana. Plant Physiol. Biochem. 49, 909-916. doi: 10.1016/j.plaphy.2011.05.013

Lukowitz, W., Nickle, T. C., Meinke, D. W., Last, R. L., Conklin, P. L., and Somerville, C. R. (2001). Arabidopsis cytl mutants are deficient in a mannose1-phosphate guanylyltransferase and point to a requirement of $\mathrm{N}$-linked glycosylation for cellulose biosynthesis. Proc. Natl. Acad. Sci. U.S.A. 98, 22622267. doi: 10.1073/pnas.051625798

Luna, E., Pastor, V., Robert, J., Flors, V., Mauch-Mani, B., and Ton, J. (2011). Callose deposition: a multifaceted plant defense response. Mol. Plant Microbe Interact. 24, 183-193. doi: 10.1094/MPMI-07-10-0149

Mukherjee, M., Larrimore, K. E., Ahmed, N. J., Bedick, T. S., Barghouthi, N. T., Traw, M. B., et al. (2010). Ascorbic acid deficiency in Arabidopsis induces constitutive priming that is dependent on hydrogen peroxide, salicylic acid, and the NPR1 gene. Mol. Plant Microbe Interact. 23, 340-351. doi: 10.1094/MPMI23-3-0340

Müller-Moulé, P., Golan, T., and Niyogi, K. K. (2004). Ascorbate-deficient mutants of Arabidopsis grow in high light despite chronic photooxidative stress. Plant Physiol. 134, 1163-1172. doi: 10.1104/pp.103.032375

Müller-Moulé, P., Havaux, M., and Niyogi, K. K. (2003). Zeaxanthin deficiency enhances the high light sensitivity of an ascorbate deficient mutant of Arabidopsis. Plant Physiol. 133, 748-760. doi: 10.1104/pp.103.026252

Munné-Bosch, S., Queval, G., and Foyer, C. H. (2013). The impact of global change factors on redox signaling underpinning stress tolerance. Plant Physiol. 161, 5-19. doi: 10.1104/pp.112.205690

Noctor, G., and Foyer, C. H. (2011). Ascorbate and Glutathione: the heart of the redox hub. Plant Physiol. 555, 2-18. doi: 10.1104/pp.110.167569

Page, M., Sultana, N., Paszkiewicz, K., Florance, H., and Smirnoff, S. (2012). The influence of ascorbate on anthocyanin accumulation during high light acclimation in Arabidopsis thaliana: further evidence for redox control of anthocyanin synthesis. Plant Cell Environ. 35, 388-404. doi: 10.1111/j.13653040.2011.02369.x

Pastori, G. M., Kiddle, G., Antoniw, J., Bernard, S., Veljovic- Jovanovic, S., Verrier, P. J., et al. (2003). Leaf vitamin C contents modulate plant defense transcripts and regulate genes that control development through hormone signaling. Plant Cell 15, 939-951. doi: 10.1105/tpc.010538

Pavet, V., Olmos, E., Kiddle, G., Mowla, S., Kumar, S., Antoniw, J., et al. (2005). Ascorbic acid deficiency activates cell death and disease resistance responses in Arabidopsis. Plant Physiol. 139, 1291-1303. doi: 10.1104/pp.105. 067686

Sanmartin, M., Drogoudi, P. D., Lyons, T., Pateraki, I., Barnes, J., and Kanellis, A. K. (2003). Over-expression of ascorbate oxidase in the apoplast of transgenic tobacco results in altered ascorbate and glutathione redox states and increased sensitivity to ozone. Planta $216,918-928$.

Scholl, R., Rivero-Lepinckas, L., and Crist, D. (1998). Growth of plants and preservation of seeds. Methods Mol. Biol. 82, 1-12. doi: 10.1385/0-89603-3910:1

Szecowka, M., Heise, R., Tohge, T., Nunes-Nesi, A., Vosloh, D., Huege, J., et al. (2013). Metabolic fluxes in an illuminated Arabidopsis rosette. Plant Cell 25, 694-714. doi: 10.1105/tpc.112.106989

Talla, S., Riazunnisa, K., Padmavathi, L., Sunil, B., Rajsheel, P., and Raghavendra, A. S. (2011). Ascorbic acid is a key participant during the interactions between chloroplasts and mitochondria to optimize photosynthesis and protect against photoinhibition. J. Biosci. 36, 163-173. doi: 10.1007/s12038-011-9000-x

Veljovic-Jovanovic, S. D., Pignocchi, C., Noctor, G., and Foyer, C. H. (2001). Low ascorbic acid in the vtc-1 mutant of Arabidopsis is associated with decreased growth and intracellular redistribution of the antioxidant system. Plant Physiol. 127, 426-435. doi: 10.1104/pp.010141

Wang, J., Yu, Y., Zhang, Z., Quan, R., Zhang, H., Ma, L., et al. (2013). Arabidopsis CSN5B interacts with VTC1 and modulates ascorbic acid synthesis. Plant Cell 25, 625-636. doi: 10.1105/tpc.112.106880

Yoshimura, K., Nakane, T., Kume, S., Shiomi, Y., Maruta, T., Ishikawa, T., et al. (2014). Transient expression analysis revealed the importance of VTC2 expression level in light/dark regulation of ascorbate biosynthesis in Arabidopsis. Biosci. Biotechnol. Biochem. 78, 60-66. doi: 10.1080/09168451. 2014.877831

Zechmann, B. (2011). Subcellular distribution of ascorbate in plants. Plant Signal. Behav. 6, 360-363. doi: 10.4161/psb.6.3.14342

Zhang, Z., Wang, J., Zhang, R., and Huang, R. (2012). The ethylene response factor AtERF98 enhances tolerance to salt through the transcriptional activation of ascorbic acid synthesis in Arabidopsis. Plant J. 71, 273-287. doi: 10.1111/j.1365313X.2012.04996.X

Zhu, Y., Du, B., Qian, J., Zou, B., and Hua, J. (2013). Disease resistance gene-induced growth inhibition is enhanced by rcd1 independent of defence activation in Arabidopsis. Plant Physiol. 161, 2005-2013. doi: 10.1104/pp.112.213363

Conflict of Interest Statement: The authors declare that the research was conducted in the absence of any commercial or financial relationships that could be construed as a potential conflict of interest.

Copyright $\odot 2016$ Lim, Smirnoff, Cobbett and Golz. This is an open-access article distributed under the terms of the Creative Commons Attribution License (CC BY). The use, distribution or reproduction in other forums is permitted, provided the original author(s) or licensor are credited and that the original publication in this journal is cited, in accordance with accepted academic practice. No use, distribution or reproduction is permitted which does not comply with these terms. 